\title{
A!
}

This is an electronic reprint of the original article.

This reprint may differ from the original in pagination and typographic detail.

Leithon, Johann; Werner, S.; Koivunen, Visa; Talebi, S. P.

\section{Price-aware Renewable Energy Management with Transmission Losses}

Published in:

44th IEEE International Conference on Acoustics, Speech, and Signal Processing, ICASSP 2019; Brighton; United Kingdom; 12-17 May 2019 : Proceedings

DOI:

10.1109/ICASSP.2019.8683219

Published: 01/05/2019

Document Version

Peer reviewed version

Please cite the original version:

Leithon, J., Werner, S., Koivunen, V., \& Talebi, S. P. (2019). Price-aware Renewable Energy Management with Transmission Losses. In 44th IEEE International Conference on Acoustics, Speech, and Signal Processing, ICASSP 2019; Brighton; United Kingdom; 12-17 May 2019 : Proceedings (pp. 8087-8091). [8683219]

(Proceedings of the IEEE International Conference on Acoustics, Speech, and Signal Processing). IEEE. https://doi.org/10.1109/ICASSP.2019.8683219

This material is protected by copyright and other intellectual property rights, and duplication or sale of all or part of any of the repository collections is not permitted, except that material may be duplicated by you for your research use or educational purposes in electronic or print form. You must obtain permission for any other use. Electronic or print copies may not be offered, whether for sale or otherwise to anyone who is not an authorised user. 


\title{
PRICE-AWARE RENEWABLE ENERGY MANAGEMENT WITH TRANSMISSION LOSSES
}

\author{
Johann Leithon ${ }^{\dagger}$, Stefan Werner ${ }^{\dagger *}$, Visa Koivunen ${ }^{\dagger}$ and Sayed Pouria Talebi ${ }^{\dagger}$ \\ ${ }^{\dagger}$ Dept. Signal Processing \& Acoustics, Aalto University, E-mails: firstname.lastname@aalto.fi \\ *Dept. Electronic Systems, Norwegian University of Science \& Tech., E-mail: stefan.werner@ntnu.no
}

\begin{abstract}
In this paper we propose a genie-aided strategy to optimize the use of renewable energy (RE) in a community of households with shared access to storage and RE generation facilities. The households are spread over a limited geographical area, and are subject to different time-varying power consumption profiles, and energy prices. We consider a finite number of RE generators and energy storage devices (ESDs), which are deployed in specific locations. The proposed strategy seeks to minimize the energy cost incurred by the participating households by optimizing the rate at which RE is consumed over time. Our model takes into account the power loss incurred in the transmission of energy from the generators to the loads. The optimization problem is cast as a non-convex quadratically constrained quadratic program, which is simplified in order to derive an approximate solution. Numerical results show that transmission losses and differences across price and load can significantly affect the optimal $\mathrm{RE}$ allocation among the households. The proposed strategy offers valuable insights for energy planning purposes and can be used to devise real-time RE management algorithms by incorporating the necessary forecasting techniques.
\end{abstract}

Index Terms - Renewable energy management, quadratic programming, transmission losses.

\section{INTRODUCTION}

The sharing of RE generation and storage facilities is becoming an attractive alternative for households with space limitations. Community solar (CS), for example, is an energy development model that is gaining popularity in the United States [1]. CS encompasses various models in which households cooperate to meet their energy requirements at lower economical and environmental costs.

Among the various CS models, one that has attracted considerable attention is Shared Solar, which consists of deploying solar PV panels at designated locations to power a number of households in their vicinities [2]. This configuration is attractive to households with restricted rooftop space or to the ones who prefer sharing capital investment.

In this paper we develop a mathematical model for energy management in a Shared Solar environment. Our framework can be used to optimize the rate at which the households consume energy from each RE production and storage center (REPSC). We take into account the power loss incurred by the wires connecting the REPSCs and the households. The power lines are modeled as real impedances, and the loss incurred in the transmission of power is modeled by using Ohm's law. With these considerations, we formulate a non-convex quadratically constrained quadratic programming

This work was supported in part by Academy of Finland under Grant 296849 and in part by Research Council of Norway. problem, for which we propose a simplification. The proposed simplification shrinks the original feasible space, and hence, the solution obtained satisfies all the constraints of the original formulation.

The proposed model leads to an RE management strategy, which can be used for energy planning purposes and to derive real-time energy management algorithms. Specifically, our framework allows us to devise clustering ${ }^{1}$ schemes to reduce capital investment by deploying a smaller number of connections between REPSCs and households. Moreover, in a real-time setting, the proposed strategy can be used to recompute the RE consumption schedules in response to updated estimates of future RE production and load. Thus, forecasting techniques can be incorporated in our framework to implement the optimization strategy in real time.

To the best of our knowledge, there are no works in the literature proposing energy management strategies for households in a Shared Solar environment, which are also aware of time-varying electricity prices, and power loss dissipated through ESDs and connecting wires. Related works in the literature include [3-6], where greedy $\mathrm{RE}$ management strategies are proposed at an individual level. Cooperative RE management strategies have been proposed in [7-22]. However, most of these strategies do not take into consideration electricity price variations across time and location, as well as the energy loss incurred by power lines connecting RE generators and loads.

Unlike existing works, we take into consideration the power loss incurred by the ESDs, and the wires connecting the REPSCs and the loads (households). As shown by our numerical results, the power loss incurred in the energy transmission can be used as a criterion for the households to choose some REPSCs over others. This result motivates the clustering of households to avoid the deployment of some of the connecting wires in the network. Transmission line power losses have been accounted for in works such as [11] and [23]. However, these works either disregard the time-varying nature of the electricity prices, or do not account for the losses incurred in the operation of ESDs charged with RE.

\section{SYSTEM MODEL}

\subsection{Loads, Planning Horizon, and RE Consumption Schedules}

We consider a set of $M$ households connected to the same power grid, and deployed across a finite area, as shown in Fig. 1. The planning horizon is $[0, S]$ where $S>0$ is an arbitrary positive real number. The power consumed by the $m$ th household at time $\tau \in[0, S]$, with $S>0$, is denoted by $L_{m}(\tau):[0, S] \rightarrow\left[0, L_{\max }\right]$, where $L_{\text {max }}$ denotes the maximum power that the household can consume. We consider a set of $N$ REPSCs, each one with an RE generator and ESD. The REPSCs are deployed across different locations. The

${ }^{1}$ For example, nearby households can be grouped to share different REP$\mathrm{SCs}$, avoiding the need for fully-connected configurations. 


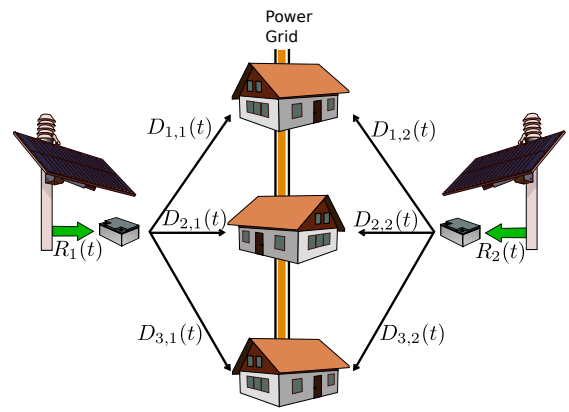

Fig. 1: Shared Solar environment with $N=2$ RE generators and $M=3$ households.

power drawn by the $m$ th household from the $n$th RE generator is $D_{m, n}(\tau):[0, S] \rightarrow\left[0, L_{m}(\tau)\right]$

\subsection{Transmission Losses, Electricity Prices, and Objective}

The power transmission losses derive from the resistance of the connecting wires. Let $\rho$ denote the resistance of the wires per unit length, hence the resistance of the wire connecting the $m$ th household and the $n$th RE generator is $\rho \operatorname{dis}_{m, n}$, where $\operatorname{dis}_{m, n}$ is the corresponding distance between the two points. The current flowing through the wire is $\frac{D_{m, n}(\tau)}{\mathrm{V}}$, where $\mathrm{V}$ is the operating voltage, assumed constant for simplicity.

The objective of the proposed algorithm is to minimize the cost incurred by all the participating households over the specified planning horizon. To achieve this, we define the objective function as the total cost spent by the households in $[0, S]$. That is,

$$
\begin{aligned}
\xi= & \sum_{m=1}^{M} \int_{0}^{S} P_{m}(t)\left[L_{m}(t)\right. \\
& \left.-\sum_{n=1}^{N}\left[D_{m, n}(t)-\rho \operatorname{dis}_{m, n}\left(\frac{D_{m, n}(t)}{\mathrm{V}}\right)^{2}\right]\right] d t
\end{aligned}
$$

where $P_{m}(\tau)$ denotes the prices offered to the $m$ th household. Price variations across location are considered to ensure generality. Our optimization framework thus seeks to reduce the cost function by designing the $D_{m, n}(\tau)$ 's.

\subsection{Energy Storage Devices}

All the ESDs in the system are characterized by:

\subsubsection{Charging/discharging losses}

The charging/discharging losses are proportional to the power charged to or discharged from the ESD. The charging/discharging efficiency rates of the $n$th ESD are respectively $\alpha_{n}$ and $\beta_{n}$, which satisfy $0<\alpha_{n} \leq 1$ and $0<\beta_{n} \leq 1$. A lossless charging (discharging) operation happens when $\alpha_{n}=1\left(\beta_{n}=1\right)$.

\subsubsection{ESD dynamics}

The energy available at the $n$th ESD is denoted by $J_{n}(\tau)$, and evolves according to:

$$
J_{n}(\tau)=J_{n}(0)+\int_{0}^{\tau}\left[\alpha_{n} R_{n}(t)-\frac{1}{\beta_{n}} \sum_{m=1}^{M} D_{m, n}(t)\right] d t
$$

where $J_{n}(0) \geq 0$ is the energy initially available in the battery, and $R_{n}(\tau)$ is the renewable power charged to the ESD, which is assumed to be within the ESD's allowed charging rate.

\subsubsection{Limited storage capacity}

The capacity of the $n$th ESD is denoted by $\Psi_{n} \in \mathbb{R}_{+}$. Therefore, the $D_{m, n}(\tau)$ 's must be such that $0 \leq J_{n}(\tau) \leq \Psi_{n}, \forall \tau$.

\subsubsection{Limited discharging rates}

Each ESD has a limited discharging rate, expressed as the maximum amount of energy that can be drawn from the ESD in each time slot. The maximum discharging rate that the $n$th ESD can handle is $q_{D, n}$ power units. Therefore,

$$
\sum_{m=1}^{M} D_{m, n}(\tau) \leq q_{D, n}, \forall \tau, \forall n .
$$

\section{PROBLEM STATEMENT}

\subsection{Decision Variables and Constraints}

The decision variables are the discharging schedules $D_{m, n}(\tau)$ which will determine the optimal RE consumption patterns. There are thus two kinds of constraints that need to be satisfied in the formulated optimization problem. The first set of constraints derives from the bounded storage capacities of the ESDs and the causality condition, according to which only RE readily available in the ESDs can be dispatched. These constraints can be stated mathematically as follows:

$0 \leq J_{n}(0)+\int_{0}^{\tau}\left[\alpha_{n} R_{n}(t)-\frac{1}{\beta_{n}} \sum_{m=1}^{M} D_{m, n}(t)\right] d t \leq \Psi_{n}, \forall \tau, \forall n$,

where $\Psi_{n}$ denotes the storage capacity of the ESD at the $n$th REPSC. Constraints (4) were obtained by using the definition of $J_{n}(\tau)$, presented in (2), and are introduced to enforce that each $J_{n}(\tau)$ is within the range $\left[0, \Psi_{n}\right]$. A second type of constraints arises if we assume that distributed RE generation needs to be used only locally, ${ }^{2}$ which means that the consumption of RE is upper bounded by the load in each household:

$$
\sum_{n=1}^{N}\left[D_{m, n}(\tau)-\rho \operatorname{dis}_{m, n}\left(\frac{D_{m, n}(\tau)}{\mathrm{V}}\right)^{2}\right] \leq L_{m}(\tau), \forall \tau, \forall m .
$$

The left-hand side of (5) represents the effective power drawn from the $m$ th household from all the $N$ REPSCs, i.e., the power obtained after transmission losses. The right-hand side of (5) denotes the power required by the $m$ th household at time $\tau$.

\subsection{Formulation and Considerations}

We formulate a mathematical problem to optimize the discharging schedules as follows:

$$
\begin{array}{cr}
\text { P0: } & \min \\
\text { s.t. } & D_{m, n}(\tau), m \in\{1, \ldots, M\}, n \in\{1, \ldots, N\} \\
\text { (3), (4), and (5). }
\end{array}
$$

\footnotetext{
${ }^{2}$ This constraint follows when no RE can be injected into the grid. In a net metering scenario, this constraint can be updated to include subscriber limits.
} 
$\mathrm{P} 0$ is a very challenging problem because of the following reasons: Its objective is not a function, but a sum of functionals. Its decision variables are not scalar or vectors, but trajectories (functions defined in continuous time). Equations (4) and (5) represent and infinite number of constraints, which must hold in all the realizations of the stochastic processes $L_{1}(\tau), \ldots, L_{M}(\tau)$ and $R_{1}(\tau), \ldots, R_{N}(\tau)$.

\subsection{Genie-Aided Solution}

We propose a method to solve $\mathrm{P} 0$ by assuming full ${ }^{3}$ knowledge of the loads and RE generation profiles across time. This genie-aided solution can be used to benchmark online strategies, and to devise realtime RE management algorithms based on forecasts. For tractability, we will introduce discretization, and determine the optimal $D_{m, n}(\tau)$ only at a finite number of points. After introducing discretization, the problem can be cast as a quadratically constrained quadratic programming problem as will be shown next.

We sample the functions $P_{m}(\tau), D_{m, n}(\tau), L_{m}(\tau)$ and $R_{n}(\tau)$, $\forall m, \forall n$, at $T>1$ equally-spaced points, and thus divide the planning horizon into $T-1$ subintervals. Let $\Delta t$ denote the sampling interval, $t \in\{1, \ldots, T\}$ be the slot index, and $\mathbf{x} \in \mathbb{R}^{M N T}$ denote the $M N T$ variables to optimize over the entire planning horizon stacked as follows: $\mathbf{x}=\left[\mathbf{x}_{1}, \mathbf{x}_{2}, \ldots, \mathbf{x}_{M}\right]^{T}$, where $\mathbf{x}_{m}=\left[\mathbf{y}_{m, 1}, \ldots, \mathbf{y}_{m, T}\right]$ and $\mathbf{y}_{m, t}=\left[D_{m, 1}(t \Delta t), D_{m, 2}(t \Delta t)\right.$, $\left.\ldots, D_{m, N}(t \Delta t)\right]$. Moreover, we denote the pricing vectors obtained after the sampling by $\mathbf{p}_{m}$, i.e., $p_{m}(t)=P_{m}(t \Delta t)$, and simplify notation by introducing the following definitions $K_{m, n} \triangleq \rho \operatorname{dis}_{m, n} \frac{1}{\mathrm{~V}}$ and $\mathbf{k}_{m}=\mathbf{1}_{T} \otimes\left[K_{m, 1}, K_{m, 2}, \ldots, K_{m, N}\right]$. Then, the objective function can be written as the following quadratic form:

$$
\xi \approx \Delta t\left[\mathbf{1}_{1, M}\left[\begin{array}{c}
\mathbf{p}_{1}^{T} \boldsymbol{\ell}_{1} \\
\vdots \\
\mathbf{p}_{M}^{T} \boldsymbol{\ell}_{M}
\end{array}\right]-\mathbf{1}_{1, M} \mathbf{P} \mathbf{x}+\mathbf{x}^{T} \mathbf{Q K} \mathbf{x}\right],
$$

with $\mathbf{P}, \mathbf{Q}$ and $\mathbf{K}$ written as follows:

$$
\begin{gathered}
\mathbf{P}=\operatorname{dg}\left(\left(\mathbf{p}_{1} \otimes \mathbf{1}_{N}\right),\left(\mathbf{p}_{2} \otimes \mathbf{1}_{N}\right), \ldots,\left(\mathbf{p}_{M} \otimes \mathbf{1}_{N}\right)\right), \\
\mathbf{Q}=\operatorname{dg}\left(\operatorname{dg}\left(\mathbf{p}_{1} \otimes \mathbf{1}_{N}\right), \operatorname{dg}\left(\mathbf{p}_{2} \otimes \mathbf{1}_{N}\right), \ldots, \operatorname{dg}\left(\mathbf{p}_{M} \otimes \mathbf{1}_{N}\right)\right), \\
\mathbf{K}=\operatorname{dg}\left(\left[\mathbf{k}_{1}, \mathbf{k}_{2}, \ldots, \mathbf{k}_{M}\right]\right),
\end{gathered}
$$

where $\operatorname{dg}(\mathbf{v})$ denotes a matrix with vector $\mathbf{v}$ in its main diagonal, and zeros elsewhere. Constraints (3) and (4) can be written in terms of $\mathbf{x}$ as follows:

$$
\mathbf{M}_{1} \mathbf{x} \preceq \mathbf{v}_{1}, \quad \mathbf{M}_{2} \mathbf{x} \preceq \mathbf{v}_{2}, \mathbf{M}_{3} \mathbf{x} \preceq \mathbf{v}_{3},
$$

for matrices $\mathbf{M}_{1}, \mathbf{M}_{2}$ and $\mathbf{M}_{3}$, and vectors $\mathbf{v}_{1}, \mathbf{v}_{2}$ and $\mathbf{v}_{3}$, which can be derived from (3) and (4), respectively. Constraints (5) can be written using the following quadratic form

$$
\mathbf{1}_{M} \mathbf{U}_{m, t} \mathbf{x}-\mathbf{x}^{T} \mathbf{V}_{m, t} \mathbf{x} \leq L_{m}(t \Delta t), \forall m, \forall t,
$$

for appropriate matrices $\mathbf{U}_{m, t}$ and $\mathbf{V}_{m, t}$. A discrete-time version of $\mathrm{P} 0$ can then be written as:

$$
\begin{array}{lrr}
\text { P1: } & \max _{\mathbf{x}} & \mathbf{1}_{M} \mathbf{P x}-\mathbf{x}^{T} \mathbf{Q K x} \\
\text { s.t. } & \text { (7) and (8). }
\end{array}
$$

In P1 we have removed the term $\Delta t \sum_{m=1}^{M} \sum_{t=1}^{T} p_{m}(t) \ell_{m}(t)$, because it does not depend on the design variable $\mathbf{x}$. Similarly, for

\footnotetext{
${ }^{3}$ This assumption does not limit the application of the proposed strategy, as forecasts can be used to replace information unavailable in a practical setting.
}

simplicity, the constant $\Delta t$ has been removed from the objective function, as it does not affect the result of the optimization. By solving P1 we can determine the optimal discharging schedules at equally-spaced time instants. The higher the sampling rate, the more information about the optimal solution can be obtained through the discrete-time formulation.

Remark: P1 is a quadratically constrained quadratic programming problem in which we want to maximize a concave function of $\mathbf{x}$. However, P1 is not a convex optimization problem because the matrices $\mathbf{V}_{m, t}$ are positive definite and preceded by a negative sign in constraints (8). To show that $\mathbf{V}_{m, t}$ is a positive definite matrix, we note that it is a diagonal matrix, since no cross-terms appear in (8), and it only has non-negative numbers because the coefficients of the quadratic terms $\left[D_{m, n}(t)\right]^{2}$ are all positive, i.e., the resistance per unit length $\rho$, the distance between the generators and the households $\operatorname{dis}_{m, n}$, and the voltage $\mathrm{V}$, are all non-negative quantities.

\subsection{Simplification}

To tackle P1, we propose the following simplified formulation:

$$
\begin{array}{cc}
\mathrm{P} 2: \max _{\mathbf{x}} \mathbf{1}_{M} \mathbf{P} \mathbf{x}-\mathbf{x}^{T} \mathbf{Q K} \mathbf{x} \\
\text { s.t. } & \text { (7) and } \\
\mathbf{1}_{M} \mathbf{U}_{m, t} \mathbf{x} \leq L_{m}(t \Delta t), \forall m, \forall t,
\end{array}
$$

which replaces (8). Note that (9) is a more stringent constraint than the original (8). As a result, this simplification will shrink the feasible space, and hence, the solution obtained for P2 will satisfy the original constraint (8).

Proposition 1. P2 can be written as a convex optimization problem.

Proof. The quadratic form $\mathbf{1}_{M} \mathbf{P x}-\mathbf{x}^{T} \mathbf{Q K x}$ is concave in $\mathbf{x}$ because QK is positive semi-definite. In P2 we thus seek to maximize a concave function. Moreover, the constraints (7) and (9) are all affine. Therefore $\mathrm{P} 2$ can be written as a convex optimization problem simply by expressing it as a minimization problem by multiplying its objective function by -1 .

\section{NUMERICAL RESULTS}

We provide numerical results to analyze the proposed strategy. Several simulation scenarios (load, RE generation profiles and prices) are considered in this section in order to illustrate the characteristics of the proposed strategy. System parameters that are used throughout this section are presented in Table 1. The optimization problems are solved by using CVX on Matlab. Throughout this section, storage capacity is measured in energy units [EU] and energy expenditure in monetary units [MU]. To simplify notation, let $D_{m}(t)$ denote the total renewable power drawn by the $m$ th household at time $t$, i.e., $D_{m}(t)=\sum_{n=1}^{N} D_{m, n}(t), \forall t$.

Table 1: System Parameters

\begin{tabular}{|l|l|}
\hline Parameter & Value \\
\hline \hline$\{T, \Delta t, M, N\}$ & $\{20,1,3,2\}$ \\
\hline$q_{D, n}$ & $\Delta t \sum_{t=1}^{T} r_{n}(t), \forall n$ \\
\hline$\left\{\alpha_{n}, \beta_{n}, \Psi_{n}, J_{n}(0)\right\}$ & $\left\{1,1, \Delta t \sum_{t=1}^{T} r_{n}(t), 0, \forall n\right\}$ \\
\hline
\end{tabular}

We start by illustrating the characteristics of the RE consumption schedule. We thus consider time-varying prices and loads, and 
fixed resistances across connecting wires of equal length. Specifically, we assume the price and load profiles illustrated in Fig. 2, and let $K_{1,1}=K_{1,2}=0.01, K_{2,1}=K_{2,2}=0.01$, and $K_{3,1}=$ $K_{3,2}=0.01$. In Fig. 2 we can see that, when the wires connecting the households and the RE generators have the same characteristics (length and resistance), the RE consumption rate is only influenced by the price variations across time and the load. When the load is above the RE generation at all times, then the optimal schedule only responds to price variations across time. When the load is below the RE generation at all times, then the optimal schedule is determined by the loads, since the consumption of RE is upper bounded by the load in each household.

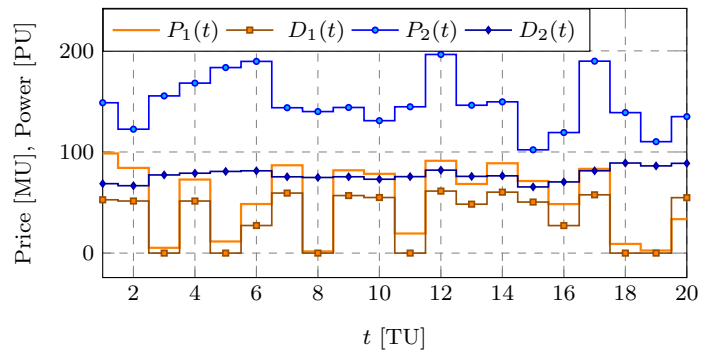

Fig. 2: Time-variation of RE consumption rate. Top: Influenced by price, when the load is above the RE generation at all times.

We now investigate the effect of the power loss factor in the RE use pattern. We thus consider constant prices, loads and RE generation profiles, but varying distances and wire resistances. Specifically, we assume the price, load and RE generation profiles illustrated in Fig. 3, and let $K_{1,1}=K_{1,2}=0.005, K_{2,1}=K_{2,2}=0.008$, and $K_{3,1}=K_{3,2}=0.01$. In Fig. 3 we illustrate both, the RE consumption rates across time, and the result of the RE allocation strategy. As seen, the RE consumption rates are nearly constant. Interestingly, since $K_{m, 1}=K_{m, 2}, \forall m$, in this scenario the share of $\mathrm{RE}$ that goes to the $m$ th household from the $n$th generator is given by the ratio $\frac{\frac{1}{K_{n, m}}}{\sum_{j=1}^{N} \frac{1}{K_{j, m}}}$.
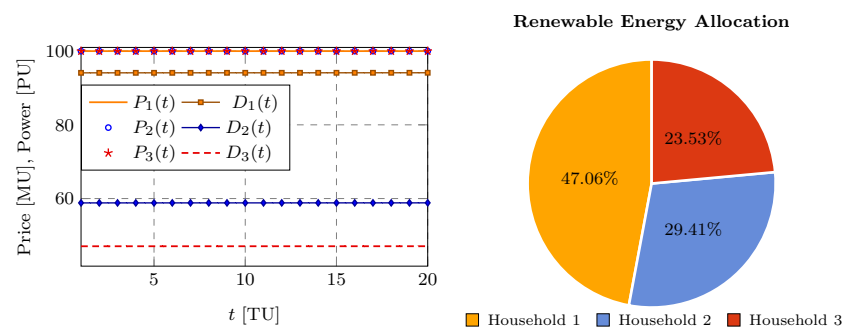

Fig. 3: Effect of power loss in RE allocation across households. Share of RE is given by $\frac{\frac{1}{K_{n, m}}}{\sum_{j=1}^{N} \frac{1}{K_{j, m}}}$. More RE is allocated to the households with better connecting power lines.

When the power loss is high, e.g., when $K_{11}=K_{12}=K_{21}=$ $K_{22}=K_{31}=K_{32}=0.02$, prices will have a reduced impact on the RE allocation policy. As seen in Fig. 4, all households get the same share of RE use, despite the price differences across locations. This result follows because the load is above the RE generation at all times, the wires connecting households and RE generators have the same characteristics (length and resistance), and the quadratic term dominates the objective function in $\mathrm{P} 0$.

As expected, prices will dominate the RE allocation policy when households and RE generators have similar characteristics, and when the power loss is not as dominant as in the scenario considered in Fig. 4. This can be seen in Fig. 5, where we considered a scenario in which prices vary across locations and connecting lines offer less than significant resistance, i.e., $K_{11}=K_{12}=K_{21}=K_{22}=$ $K_{31}=K_{32}=0.002$.
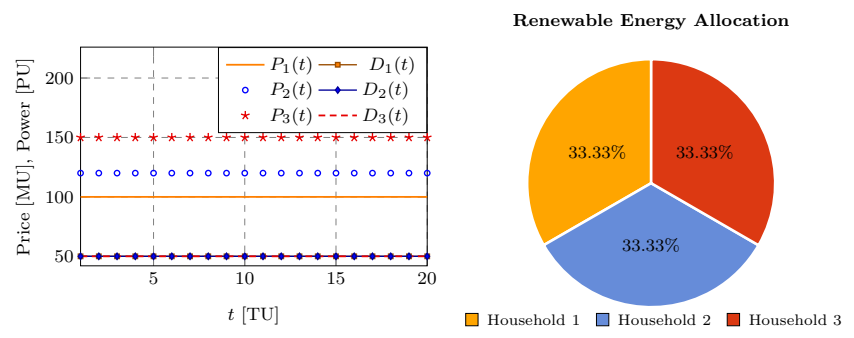

Fig. 4: Despite price differences accross households, the RE allocation is even, following poor power connecting lines.
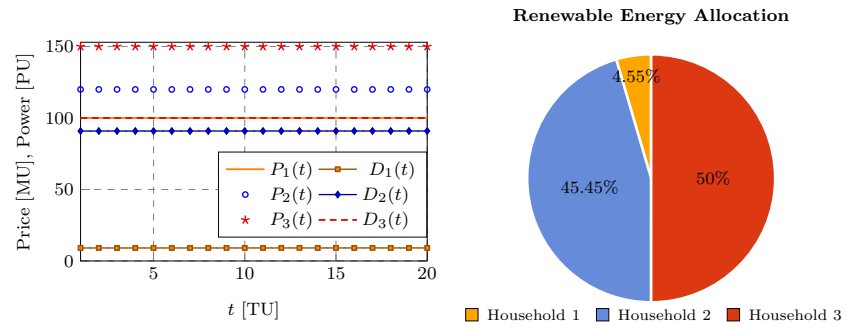

Fig. 5: With highly efficient connecting lines, the RE allocation policy is mainly determined by the price differences across households.

\section{CONCLUSIONS}

We have proposed an energy management strategy which seeks to minimize the cost incurred by a cooperating group of households over a finite planning horizon. The households share access to a group of RE generators and ESDs. In our framework we have considered the distance-dependent power loss incurred when transmitting energy from the RE generators to the loads. We have cast the optimization problem as a non-convex quadratically constrained quadratic programming problem and proposed a solution through discretization and relaxation. We have presented numerical results to illustrate the characteristics of the proposed solution. Through simulations, we have shown that the RE consumption rate depends on the price variations across time, the loads, and the characteristics of the power lines connecting the households and the RE generators. The proposed strategy can be used for energy planning purposes and to benchmark and devise real-time energy management algorithms by incorporating forecasting techniques to estimate future RE generation and power consumption. It can also be used for establishing energy cooperation clusters of households so as to reduce capital expenditure, i.e., the cost incurred in the deployment of transmission lines and RE production centers. 


\section{REFERENCES}

[1] D. Feldman, A. M. Brockway, E. Ulrich, and R. Margolis, "Shared Solar: Current Landscape, Market Potential, and the Impact of Federal Securities Regulation," Tech. Rep. NREL/TP-6A20-63892, 2015. [Online]. Available: https://www.nrel.gov/docs/fy15osti/63892.pdf

[2] P. Augustine and E. McGavisk, "The next big thing in renewable energy: Shared solar," The Electricity Journal, vol. 29, no. 4, pp. $36-42,2016$.

[3] S. Grillo, M. Marinelli, S. Massucco, and F. Silvestro, "Optimal management strategy of a battery-based storage system to improve renewable energy integration in distribution networks," IEEE Trans. Smart Grid, vol. 3, no. 2, pp. 950-958, June 2012.

[4] J. Leithon, S. Sun, and T. J. Lim, "Demand response and renewable energy management using continuous-time optimization," IEEE Transactions on Sustainable Energy, vol. 9, no. 2, pp. 991-1000, April 2018.

[5] J. Leithon, T. J. Lim, and S. Sun, "Online demand response strategies for non-deferrable loads with renewable energy," IEEE Transactions on Smart Grid, vol. 9, no. 5, pp. 52275235, Sep. 2018.

[6] V. Pilloni, A. Floris, A. Meloni, and L. Atzori, "Smart home energy management including renewable sources: A qoedriven approach," IEEE Trans. Smart Grid, vol. PP, no. 99, pp. $1-1,2017$.

[7] G. Ye, G. Li, D. Wu, X. Chen, and Y. Zhou, "Towards cost minimization with renewable energy sharing in cooperative residential communities," IEEE Access, vol. 5, pp. 11 688-11699, 2017.

[8] Z. Zhang, N. Rahbari-Asr, and M. Y. Chow, "Asynchronous distributed cooperative energy management through gossipbased incremental cost consensus algorithm," in 2013 North American Power Symposium (NAPS), Sept 2013, pp. 1-6.

[9] I. Stoyanova, M. Biglarbegian, and A. Monti, "Cooperative energy management approach for short-term compensation of demand and generation variations," in 2014 IEEE Int. Systems Conf. Proceedings, March 2014, pp. 559-566.

[10] A. C. Luna, N. L. Diaz, M. Graells, J. C. Vasquez, and J. M. Guerrero, "Cooperative energy management for a cluster of households prosumers," IEEE Trans. Consumer Electronics, vol. 62, no. 3, pp. 235-242, August 2016.

[11] F. Mangiatordi, E. Pallotti, D. Panzieri, and L. Capodiferro, "Multi agent system for cooperative energy management in microgrids," in 2016 IEEE 16th Int. Conf. on Environment and Electrical Engineering (EEEIC), June 2016, pp. 1-5.

[12] K. Rahbar, C. C. Chai, and R. Zhang, "Energy cooperation optimization in microgrids with renewable energy integration," IEEE Trans. Smart Grid, vol. PP, no. 99, pp. 1-1, 2016.

[13] H. Dagdougui, A. Ouammi, L. Dessaint, and R. Sacile, "Global energy management system for cooperative networked residential green buildings," IET Renewable Power Generation, vol. 10, no. 8, pp. 1237-1244, 2016.

[14] Z. Wang, C. Gu, F. Li, P. Bale, and H. Sun, "Active demand response using shared energy storage for household energy management," IEEE Trans. on Smart Grid, vol. 4, no. 4, pp. 18881897, Dec 2013.
[15] I. Atzeni, L. G. Ordez, G. Scutari, D. P. Palomar, and J. R. Fonollosa, "Cooperative day-ahead bidding strategies for demand-side expected cost minimization," in 2013 IEEE International Conference on Acoustics, Speech and Signal Processing (ICASSP), May 2013, pp. 5224-5228.

[16] A. Chi, J. Rajasekharan, J. Lundn, and V. Koivunen, "Demand response for renewable energy integration and load balancing in smart grid communities," in 2016 24th European Signal Processing Conf. (EUSIPCO), Aug 2016, pp. 1423-1427.

[17] J. Rajasekharan and V. Koivunen, "Optimal energy consumption model for smart grid households with energy storage," IEEE Journal of Sel. Topics in Signal Processing, vol. 8, no. 6, pp. 1154-1166, Dec 2014.

[18] W. Tushar, J. A. Zhang, C. Yuen, D. B. Smith, and N. U. Hassan, "Management of renewable energy for a shared facility controller in smart grid," IEEE Access, vol. 4, pp. 4269-4281, 2016.

[19] Y. Zhang, N. Rahbari-Asr, J. Duan, and M. Y. Chow, "Dayahead smart grid cooperative distributed energy scheduling with renewable and storage integration," IEEE Trans. on Sustainable Energy, vol. 7, no. 4, pp. 1739-1748, Oct 2016.

[20] M. Cabras, V. Pilloni, and L. Atzori, "A novel smart home energy management system: Cooperative neighbourhood and adaptive renewable energy usage," in 2015 IEEE International Conference on Communications (ICC), June 2015, pp. 716721.

[21] B. Li, T. Chen, X. Wang, and G. B. Giannakis, "Real-time energy management in microgrids with reduced battery capacity requirements," IEEE Transactions on Smart Grid, pp. 1-1, 2018.

[22] J. Leithon, S. Werner, and V. Koivunen, "Cooperative renewable energy management with distributed generation," in 2018 26th European Signal Processing Conference (EUSIPCO), Sep. 2018, pp. 191-195.

[23] H. K. Nunna, A. M. Saklani, A. Sesetti, S. Battula, S. Doolla, and D. Srinivasan, "Multi-agent based demand response management system for combined operation of smart microgrids," Sustainable Energy, Grids and Networks, vol. 6, no. Supplement C, pp. 25 - 34, 2016. 